\title{
Noise Reduction \& Gate Plug-ins in Audio Mixing Process
}

\author{
Yoemun Yun \\ Dept. of Applied Music, Chungwoon University \\ Daehakgil-25, Hongseong, Chungnam, South Korea \\ hippie740@chungwoon.ac.kr
}

\begin{abstract}
Early gates were based on an electronic switch that was only turned on or off according to the input signal level. Variety digital noise reductions are in both hardware and software form and these are far more detailed than gates or expanders and generally work by first separating the incoming signal into a large number of slit frequency bands. Noise has been usually added to an electrical signal there is no perfect way to remove it, although there are digital process that are effective at significantly reducing the level of random noise. However, it can be good practice to assume that any notise engineer collect along the way is going to be a permanent part of the recording and that alt possible ways should be taken to minimize it. This means that engineer should alyeays optimize gain structure, un-route unused mix channels and take all other practicat measures to reduce noise at the source. Even though engineers try to do the best efforts, some noise can be remaining in signal. It is physical matter but the best way is to less unwanted'noise.
\end{abstract}

Keywords: Gate, Pink noise, White hbise, Threshold, Noise reduction, Hysteresis

\section{Introduction}

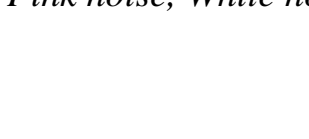

Usually, noise is a problemin all recording situations, and is happened caused by electrical circuit hiss, rolling magnetic tape hiss, errors in digital quantizing, poor screening, and amplifier ground loops and so on. There are also additional acoustic noises during the recording sessions caused by turning music papers, squeaking chairs, microphone spillage from other instruments, audience noise, wind and traffic. There are two different kinds of noises. One is pink noise. It is a type of random noise which has a constant amount of energy in each octavg band. Pink noise can be made from white noise by passing it through a filter with a 3 decioel per octave roll-off. The other is white noise. It is a special type of random noise where the energy content is the same at each frequency. True white noise would have energy extending from DC, or zero frequency, to infinitely high frequency [1]. These are always tricky parts and take time how to deal with all the noises in good way during the audio engineering and music productions.

\section{Noise Reduction}

From the sound separation point of view, the most important difference between singing and speech is the nature of other concurrent sounds. In a real acoustic environment, speech is usually contaminated by interference that can be harmonic or non-harmonic, narrowband or broadband. Interference in most cases is independent of speech in the sense that the spectral contents of target speech and interference are uncorrelated. For recorded singing voice, 
however, it is almost always accompanied by musical instruments that in most cases are harmonic, broadband, and are correlated with singing since they are composed to be a coherent whole with the singing voice [2].

Sound travels in nature. Noise is usually caused around the corner between the walls and ceilings of venues. Noise reduction is a general concern for changes in distance from a source, changes in sound absorption within a room, and sound transmission though walls. The most general form of Noise reduction is below,

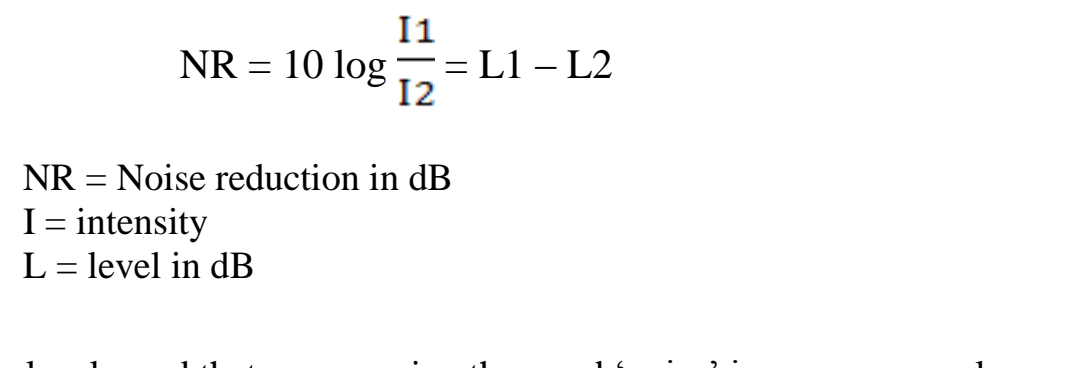

NR is the change in levels, and that we are using the yord 'noise' in a very general sense to include sounds if all kinds.

\subsection{Noise reduction outdoors}

The base of idea is the audio's keycharacteristies that many audio streams can be mixed into the stream equivalent to just one stream in size. When various sounds are audible, we can hear some of the loudest sounds. This is because the sounds having different frequency counterbalance each other. With other media such as video, text, we can't see these mixing effects. [3]

This can be calculated as the difference in levels, NR, between the two locations. NR for spherical spreading is calculated using the equation below.

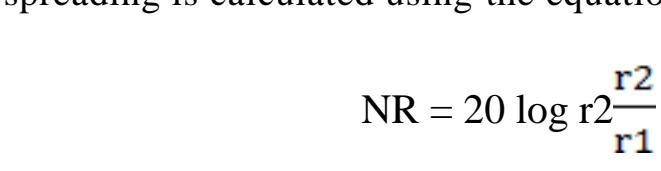

$N R=$ Noise reduction in $\mathrm{dB}$

$\mathrm{R}=$ distance from source.

This provide a method for calculating NR by knowing only the distance of concern. It is not especially mportant to know which is $\mathrm{r} 1$, and which is $\mathrm{r} 2$. Rather, if engineer moves from the closer location to the farther location, sound pressure level will decrease by the absolute value of $\mathrm{NR}$ in $\mathrm{dB}$. Conversely, if engineer moves from farther to closer, sound pressure level will increase. Because noise reduction is $6 \mathrm{~dB}$ per doubling of distance $(6 \mathrm{~dB} / \mathrm{dd})$, when the distance is doubled, $2 \mathrm{r} 1=\mathrm{r} 2$. So,

$$
\mathrm{NR}=20 \log \mathrm{r} 2 \frac{\mathrm{r} 2}{\mathrm{r} 1}=20 \log 2=6 \mathrm{~dB}
$$

\subsection{Masking noise}

Using masking effects is one of good way to eliminate noises. Unless the noise is intense and unwanted, it can be considered that the noise will only be obtrusive during quiet sections of the music as the rest of the time it will be masked by the strong signal. 
The rapid advancement of computer technologies contributed to make the speed of processor. There appeared more Internet-users, protocols and programs requiring considerable throughput. There has always been and will continue to be some kind of effort to minimize required computer processing capacity and network usage in order to save costs. [3]

Placing microphone on the right spot is hard to treat because periods of silence are rare, but separate tracks within a multi-track recording generally have some periods of silence, such as space between vocal verse and chorus, and instrument solo, including pauses between words and phrases as well as longer gaps during instrumental passages. If the signal level could be turned down during these periods, the backgrøund hiss would also be turned down so that the entire sound is not affected by noise [4].

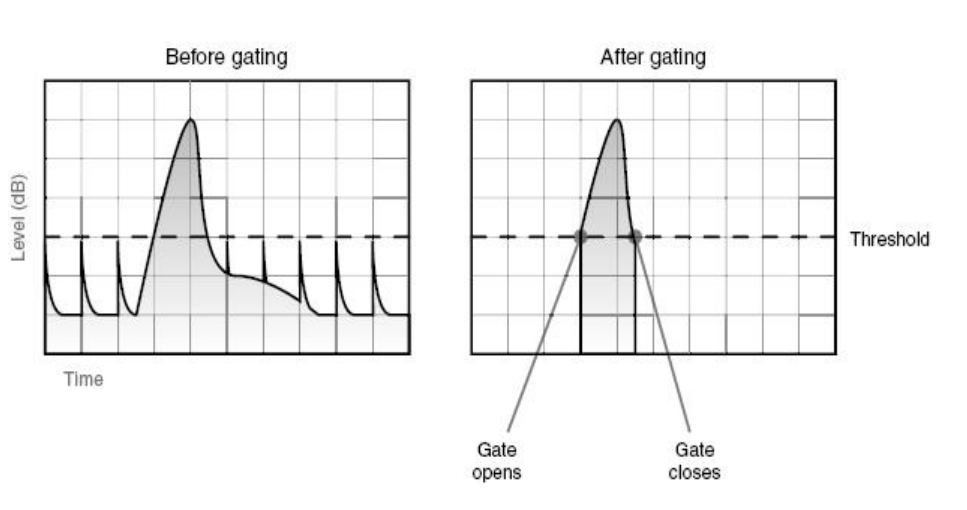

Fígure 1. Gate with Threshold

However, doing the above way to avoid unwanted noise on every track by using faders is inconvenient, even using fadeautomation, so this procedure is performed with an automatic electronic muting device call a gate or noise gate. Early gates were based on an electronio switch that as only turned on or off according to the input signal level.

There is a threshold on the gate set by the user, similar like a compressor, but on a gate the electronic switch operates to mute the signal when the signal falls below the threshold. A compressor processes signals above the threshold while a gate treats signals below it. Figure-1 illustrates how gate threshold system operates, and it has some soundreduction LED indicator to show if it is open and closed [4].

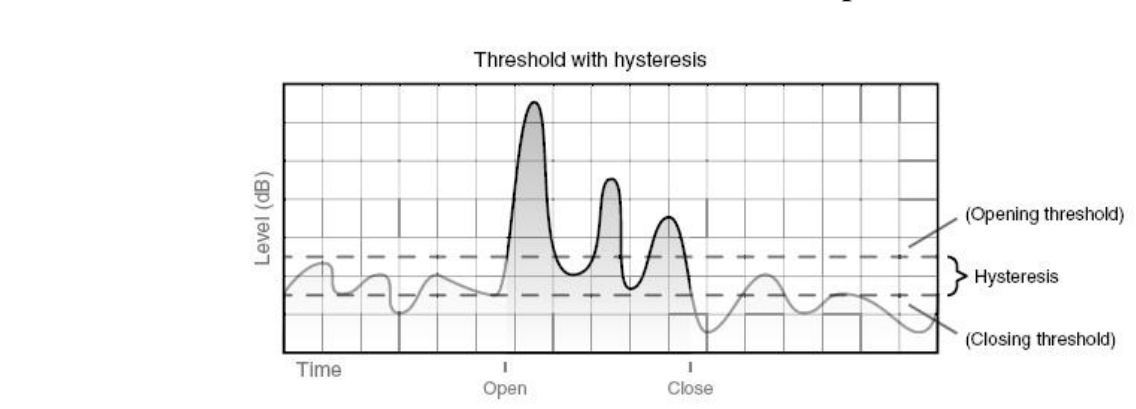

Figure 2. Opening \& Closing Threshold

A gate has a simple switch that is either on or off with no in-between state, but in this case, quiet sounds are in danger of being muted along with the noise. Also, sounds with 
natural decays are kind of weak to this effect. Advanced or more detailed gate action with variety functions is needed.

\subsection{Attack and release}

To avoid the problem of chopping off low-level sounds, studio gates are fitted with a release time control so that the sound is faded out over a period of time set by the user instead of simply being switched off when the signal falls below the threshold. This represents a dramatic improvement and prevents the noise from being turned on and off too suddenly. An effect which is offensive in most instances than the continuous background noise the gate is trying to hide.

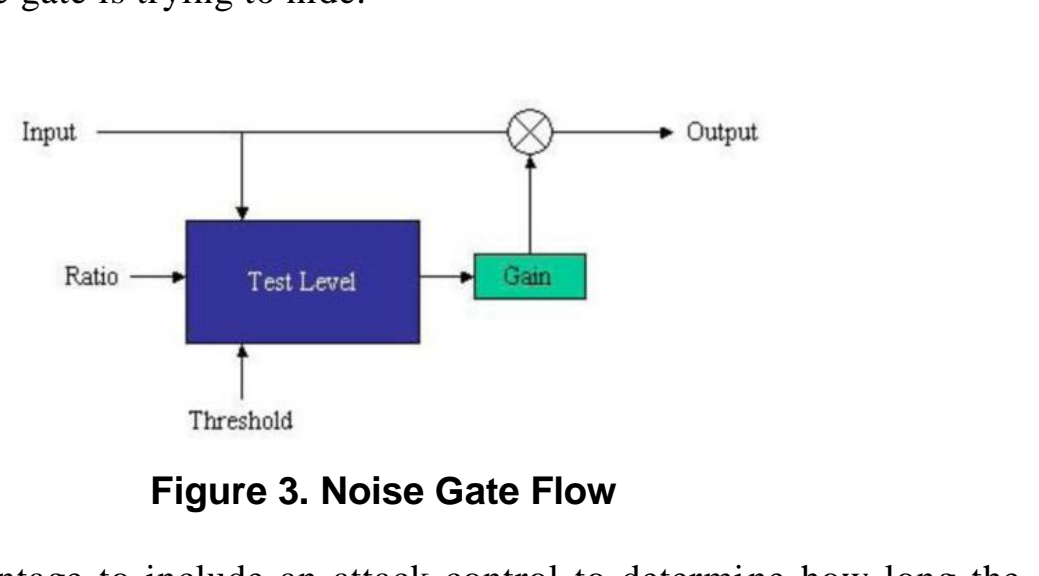

It is also an advantage to include an attack control to determine how long the gate takes to open fully once the input signal exceeds the pre-determined threshold. If the gate opens to quickly, low-frequency sounds may be distorted due to the gate operating during through a cycle, which can produce unwanted sound like click. This problem can be solved by slowing down the attack time.

Also, a soupd like the beat of a snlare drum, on the other hand, will be snatched of its impact if the gate opens tooslowly, so the solution of the problem has to be made for very fast operation in these situations.

\subsection{Range and Hold time}

Gate units including some compressors have an extra control called range or attenuation, which lets a certain amount of signal to pass even though the gate is closed. This might be useful during a recording session in which the room ambience is required in the mix, although at a lower level. By using the gate to attenuate this by a few decibels rather than switching it, the ambience can be allowed through during pauses at a more wanted level.

Some of advanced gates might come across the hold setting. Hold function stops the gate entering its release phase for a pre-determined time after the input falls below the threshold. A fast release setting can be used to abruptly gate reverb or room ambience in order to create the well-known gated drum sound, a shorter hold setting may be useful to prevent the gate from noise, which occurs when the input signal constantly changes up and down in level, through selecting a longer release time is also effective against the noise in specific situations [5]. 


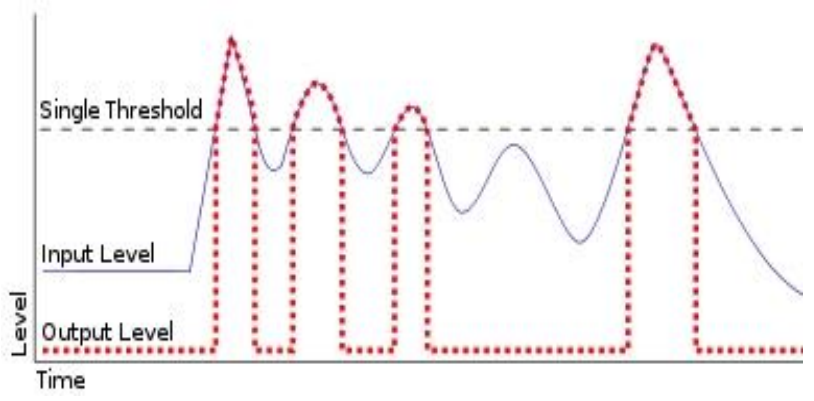

Figure 4. Gate without Hysteresis

\subsection{Hysteresis and Side chains}

Chattering noise can be a worst problem when the sound which is $\mathrm{being}$ gated has a slowly fluttering decay waveform, so the best way using gates in that case are designed to exhibit a hysteresis effect which means that the signal must fall a few decibels below the threshold at which the gate opens before the gate starts to close again. This function is the same meaning as having two thresholds one for opening the gate and a lower one for closing it. In the real practice, it means that the inputsignal has to waver in level by more than retriggering can occur.

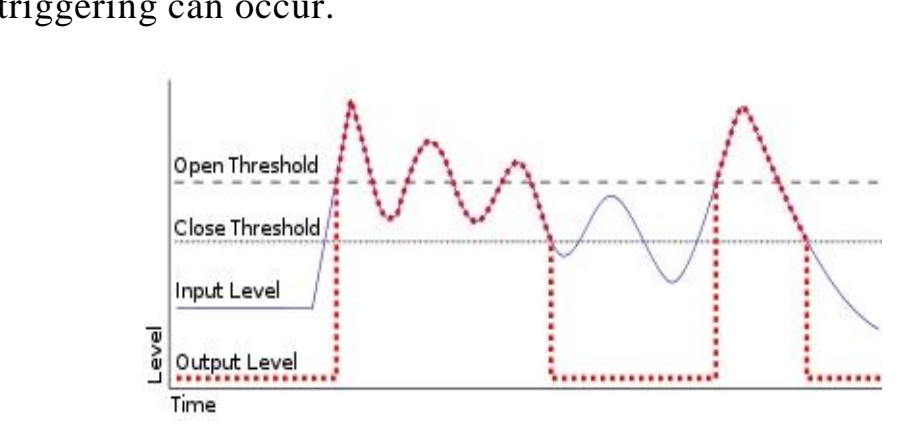

Figure 5. Gate with Hysteresis

The basic spread spectrum technique, on the other hand, is designed to encode a stream of information by spreading the encoded data across as much of the frequency spectrum as possible. This allows the signal reception, even if there is interference on some frequencies [6].

Singing voice removal algorithm consists of power comparison between each channel of the stereo signal and inter-channels differential signal and then spectrally removed at each singing voice frequency bin when larger than threshold value respectively [3].

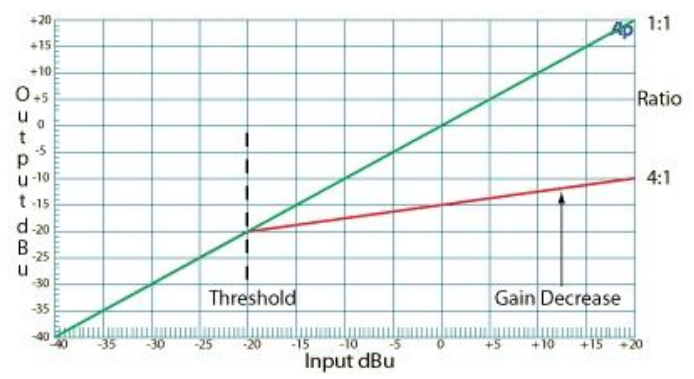

Figure 6. Compressor graph 
Like compressor, the gate has a side-chain circuit which measures the level of the incoming signal and compares it to the threshold set by the user. When the threshold is exceeded, the circuit generates a control signal to open the gate at a rate set by the attack control. When the signal falls below the threshold, the gate closed according to the setting of the hold and release controls.

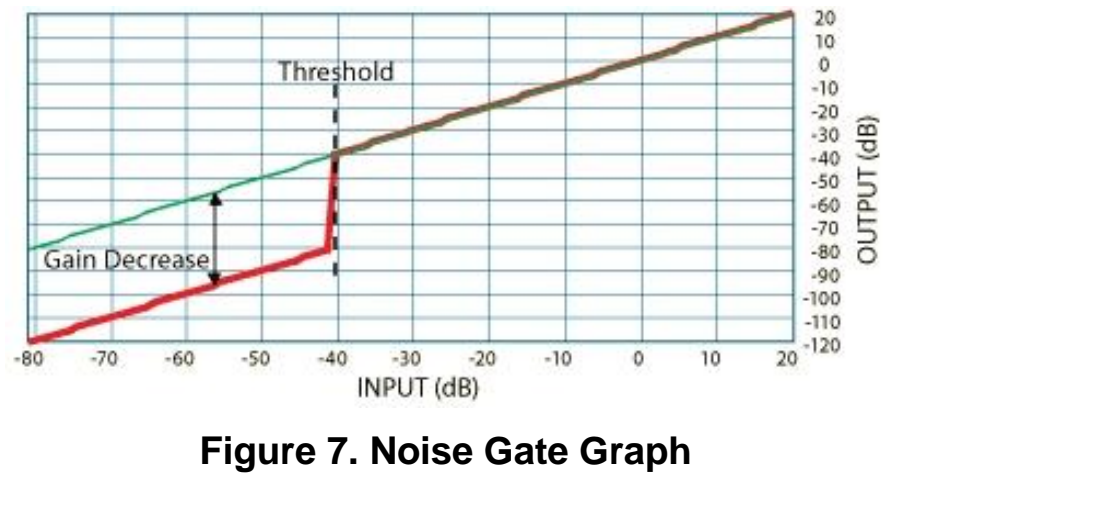

Like the compressor, there is link function that a two-channel gate has a linking switch that both channels switch on and off at the same time when stereo channel is being processed, even though the signat is louder in one channel than in the other.

A time delay element is used to provide such ambience effects, and a $\pm 90^{\circ}$ phase shifter is needed to present spadiousness effects. Assuming that the distance from front loudspeakers to the wall is about 2 meters and the distance from rear loudspeakers to the wall is about 1 meter, a time delay of $12 \mathrm{~ms}$ is applied to the surround channels [2].

Like the compressor, this is necessary to prevent the stereo image from shifting signals, which occurs if both channels operate separately.

Not the only audio data size, the CPU load in decompressing the compressed audio data should be also considered. The data volume and CPU load from data decompression would be increased rapidly [3].

In real world, there are few applications that require stereo operation, other than that involved in gating the output from a stereo reverb or reverb chamber to create a gated reverb effect especially for snare, kick and toms.

\subsection{Digital Noise Réduction}

Nowadas, digital noise reduction systems have been designed by many manufacturers.

The input is first partitioned into spectrally homogeneous portions detecting signifieant spectral changes. Then, from energy comparison between differential signals between stereo AR (all recorded) signal and each channel signals in frequency domain, the presence or absence of singing voice were determined. The proposed systems were implemented in DSP board. And input AR stereo signal, extracted MR (music recorded) signal from DSP output, and extracted singing voice could be listened in real-time by multichannel audio interface cooperated with DSP board [2]. Variety digital noise reductions are in both hardware and software form and these are far more detailed than gates or expanders and generally work by first separating the incoming signal into a large number of split frequency bands. Some systems can split the audio into over 500 different bands, where each band is equipped with the digital equivalent of its own expander [7].The signal within that band is attenuated when the signal within the band 
falls to a threshold set above the noise floor. This can reduce the side-effects introduced by simple gating or expansion because only those bands in which nothing is happening are attenuated.

Instead of breaking a signal down into individual samples, the parity coding method breaks a signal down into separate regions of samples and encodes each bit from the secret message in a sample region's parity bit. [6]. The signal is unaffected in any band showing activity above the threshold as is the noise within those bands, but in most cases the wanted signal masks any noise happening within the same frequency range.

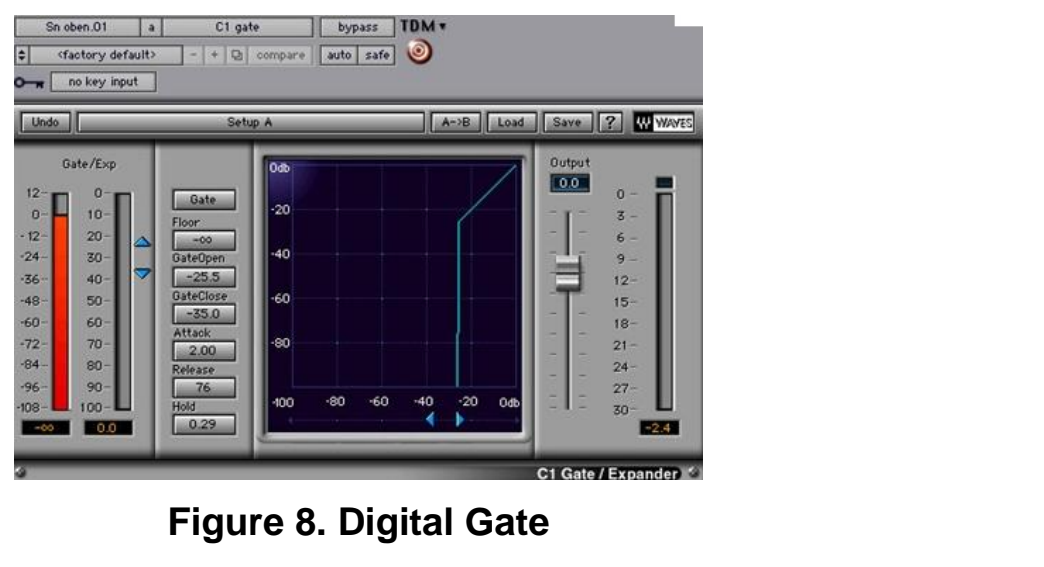

When using the function, this ho very important to figure out how to set several hundred different thresholds to the right level. If the noise spectrum is flat, having a common control is fine.

If audio content having fewer channels than can be provided by a target system is available, the target atdio system cannot take full advantage of it. Therefore, in order to utilize such audio content it is hecessary to use an upmixing method that converts mono or stereo audio formats into a multi-channel audio format suitable for such a system [8].

But in real situation, the noise energy usually increases at low frequencies, and with certain sources there may be peaks in the noise spectrum on the unit.

Before or after the recording of the wanted material, some systems depend on the presence of a short section of background noise and an absence of signal directly. A short section of this noise is selected, usually less than a second in length, and the software is required to learn the noise factors 19 .

Because noise does not have a constant spectrum, some multiband noise-reduction systems have limited improvement in noise performance without audible side effects in partly. These change throughout a recording as noise sources, and are just faded in and out of the mix.

\section{Conclusion}

Some advanced approaches are to use a system than can mathematically differentiate between unwanted noise and wanted signal on a continual basis so that the multi bands thresholds are updated several times a second especially in digital noise gate.

In order to utilize such audio content, it is necessary to use an upmixing method that converts mono or stereo audio formats into a multi-channel audio format suitable for such a system [8]. Systems such as gate and compressor do not require an isolated noise 
sample to analysis, which is an advanced when users operate tracks which all of the recordings have been topped and tailed to remove all tracks of noise before the starts and after the ends of the tracks. By constantly adapting to the changing noise spectrum these systems can provide around twice as much as reduction before side-effects become apparent for better sound.

\section{References}

[1] G. D. White, “The Audio Dictionary, Second Edition”, University of Washington Press, (1991).

[2] H. Kim, T.H. Kim and J. S. Park, "A Real Time Singing Voice Removal System Using DSP and Multichannel Audio Interface”, International Journal of Multimedia and Ubiquitous Engineering, vol.) 7, no. 2, (2012).

[3] Y. M. Kim, "The Audio Control using TDM(Tree-structural Distributed-terminal Mixing) in the Webcast", International Journal of Multimedia and Ubiquitous Engineering, vol. 1, no. 4, (2006).

[4] D. Franz, "Recording and Producing in the Home Studio", Berklee Pres., (2004).

[5] R. Izhaki, Audio Concepts: The Usage of Gates Explained, Focal Press, (2011).

[6] P. Dutta, D. Bhattacharyya and T. Kim, "Data Hiding in Audio Signal: A Relview" International Journal of Database Theory and Application, vol. 2, no. 2, (2009).

[7] K. C. Pohlmann, "Principles of Digital Audio", Fourth Edition, McGraw Hill, (2000).

[8] C. J. Chun, Y. G. Kim, J. Y. Yang and H. K. Kim, "Real-Time Conversion of Stereo Audio to 5.1 Channel Audio for Providing Realistic Sounds", Internationa Journal of Signal Processing, Image Processing and Pattern Recognition, vol. 2, no. 4, (2009).

[9] T. E. Rudolph and V. A. Leonard, "Recording in the Digital World", (2001).
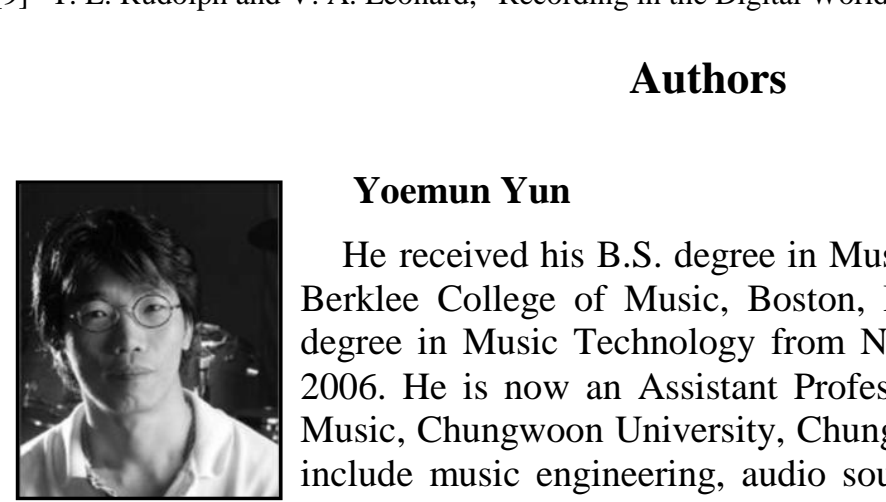

\section{Yoemun Yun}

He recerved his B.S. degree in Music Production \& Engineering from Berklee College of Music, Boston, MA, USA in 2004, and his M.S. degree in Music Technology from New York University, NY, USA in 2006. He is how an Assistant Professor in the Department of Applied Music Chungwoon University, Chungnam, Korea. His research interests include music engineering, audio sound design, analog-to-digital audio conversion, electronic music, and computer music composition. 